\title{
Temperature Dependence of Limiting Fluorescence Anisotropy of POPOP in Cellulose Acetate Film*
}

\author{
A. Kawski, A. Kubicki, and I. Weyna \\ Luminescence Research Group, Institute of Experimental Physics, \\ University of Gdańsk, Poland \\ I. Janić \\ Institute of Physics, University of Novi Sad, Yugoslavia \\ Z. Naturforsch. 40a, 559-561 (1985); received December 12, 1984
}

\begin{abstract}
The effect of temperature $(103 \mathrm{~K}<T<303 \mathrm{~K})$ upon the limiting fluorescence anisotropy $r_{0}$ of POPOP was investigated in a cellulose acetate film. A slow increase in $r_{0}$ was observed when reducing the temperature. Based on the Jabłoński theory, the frequency of the torsional vibrations of POPOP was determined to be $\omega=1.3 \times 10^{12} \mathrm{~s}^{-1}$. The depolarization due to these torsional vibrations was found to occur immediately following excitation during the thermal relaxation of the luminescent centre, thus somewhat lowering the value of the fundamental fluorescence anisotropy $r_{\mathrm{f}}$ to the limiting $r_{0}$ value.
\end{abstract}

\section{Introduction}

The effect of temperature on the limiting fluorescence anisotropy $r_{0}[1,3]$, in frozen or rigid solutions has been the object of numerous investigations [5-15]. Nevertheless, the results obtained so far cannot be considered as satisfactory in view of the following factors that should be always taken into account when measuring $r_{0}(T)$ :

a) Short- and long-wave absorption bands should be well separated to avoid simultaneous excitation in two bands,

b) the change of the solution temperature is accompanied by a change in the dielectric constant and the refractive index, which results in a shift of the absorption and fluorescence bands,

c) phosphorescence or prolongated fluorescence are undesired,

d) the mean lifetime $\tau$ of the luminescent molecule should not vary in the relevant temperature range.

The aim of the present paper is to investigate the behaviour of $r_{0}$ as a function of temperature for POPOP in a rigid solvent - a cellulose acetate film - with the above mentioned conditions being taken into account. The POPOP molecule has a

\footnotetext{
* Carried out under Research Project MR.I.5.
}

Reprint requests to Prof. Dr. Alfons Kawski, Instytut Fizyki Doświadczalnej, Uniwersytet Gdański, ul. Wita Stwosza 57, 80-952 Gdańsk, Poland. relatively low electric dipole moment $\left(\mu_{\mathrm{g}}=2.22 \mathrm{D}\right.$ and $\left.\mu_{\mathrm{e}}=3.3 \mathrm{D}[16]\right)$ and the transition moment coincides with the long molecular axis [17].

\section{Experimental}

POPOP $\left(5 \cdot 10^{-5} \mathrm{M}\right)$ in a thin cellulose acetate film (to eliminate reabsorption and secondary fluorescence) was placed between quartz plates in a cryostat of stainless steel ( 77 to $350 \mathrm{~K}$ ). The sample was excited from the front wall at an angle of $45^{\circ}$ $\left(\lambda_{\text {exc }}=385 \mathrm{~nm}\right)$. Before each measurement of $r_{0}$ the temperature was established by means of a temperature controller type 650 UNIPAN $( \pm 1 \mathrm{~K})$. The fluorescence anisotropy was measured using a sensitive self-recording polarimeter described in our previous paper [18].

\section{Results and Discussion}

Figure 1 shows the relative fluorescence anisotropy $\left(r_{\mathrm{f}}-r_{0}\right) / r_{\mathrm{f}}$ of POPOP as function of the temperature $T$. As has been observed, the limiting fluorescence anisotropy $r_{0}$ gradually grows when lowering $T$. Jabłoński [19-21] obtained the following expression for the temperature dependence of the limiting fluorescence anisotropy $r_{0}$ :

$$
r_{0}(T)=r_{\mathrm{f}}\left(1-\frac{3}{2} u(T)\right)^{2},
$$

0340-4811 / 85 / 0600-0559 \$01.30/0. - Please order a reprint rather than making your own copy. 


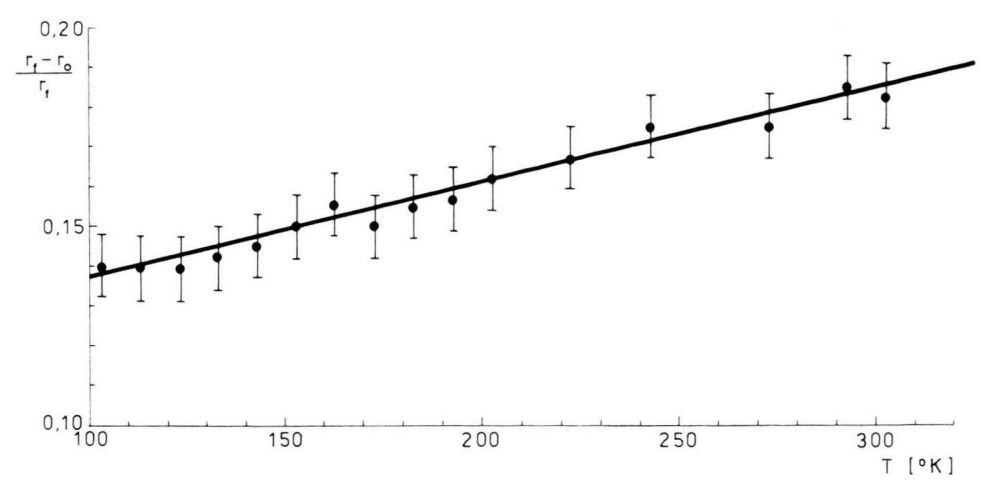

Fig. 1. Dependence of $\left(r_{\mathrm{f}}-r_{0}\right) / r_{\mathrm{f}}$ on temperature for POPOP in cellulose acetate film according to (4). where $u(T)=\left\langle\sin ^{2} \gamma\right\rangle \approx \gamma^{2}=2 \delta ; \delta$ is the dispersion of the angle of torsional vibrations along one of the two axes of the rigid molecule (torsional vibrations about the third axis, which is parallel to the direction of the absorption oscillator, do not depolarize the fluorescence light). The value of $\delta$ depends on the temperature and on the moment of torsions acting on the molecule

$$
\|=I \omega^{2} \text {, }
$$

where $\omega / 2 \pi$ is the frequency of the torsional vibrations and $I$ the moment of inertia of the molecule.

Assuming that for small amplitudes the torsional vibrations [22] of the three axes of inertia can be regarded as independent, Jabłoński [19] has given the following approximate expression for this dispersion $(k T \gg \hbar \omega)$ :

$$
u(T)=2 \delta=\frac{\hbar}{I \omega}+\frac{2 k T}{I \omega^{2}} .
$$

Since $u \ll 1$, (1) may be replaced by the following approximate formula, with (3) being taken into account:

$$
\frac{r_{\mathrm{f}}-r_{0}}{r_{\mathrm{f}}}=A+B T,
$$

where

$$
A=\frac{3 \hbar}{I \omega}
$$

and

$$
B=\frac{6 k}{I \omega^{2}} \text {. }
$$

[1] On excitation of an isotropic fluorescent solution by linearly polarized light, the fluorescence anisotropy is defined by [2]

$$
r=\frac{J-J_{\perp}}{J}=\frac{3 J}{2 J}-\frac{1}{2}=\frac{2 P}{3-P},
$$

where $J=J+2 J_{\perp}$ is the sum of all three components, $J$ and $J_{\perp}$ denote the fluorescence com-
In the case investigated, the angle $\gamma$ between the mean direction of the position equilibrium and the instantaneous direction amounts to $8.9^{\circ}$ and $5.35^{\circ}$ for $T=293 \mathrm{~K}$ and $T=103 \mathrm{~K}$, respectively, for $\omega$ determined below. Thus, the approximation given by (4) holds true.

As can be easily seen, $u$ does not vanish when the temperature tends to $0 \mathrm{~K}$. However, the formulas (3) and (4) are true only for $k T \gg \hbar \omega$, and therefore the value $A$ can not be evaluated from the extrapolation $r_{0}(T)$ for $T \rightarrow 0 \mathrm{~K}$. Plotting the left-hand side of (4) as a function of $T$ for $r_{\mathrm{f}}=0.4$ one obtains a straight line with the slope $B$ (Figure 1). The moment of inertia $I$ can be calculated from the known structure of the molecule. The mean moment of inertia with respect to the axis perpendicular to the long axis of the POPOP molecule results to be $I=19.7 \times 10^{-44} \mathrm{~kg} \mathrm{~m}^{2}$ and is considerably smaller than that determined from the rotational depolarization in liquid solvent [24]. The vlue of $B$ (Fig. 1) obtained is $2.4 \times 10^{-4} \mathrm{~K}^{-1}$ and, hence, from (6) $\omega=1.3 \times 10^{12} \mathrm{~s}^{-1}$ and from (2) $\mathscr{M}=3.3 \times 10^{-19}$ $\mathrm{m}^{2} \mathrm{~kg} \mathrm{~s}^{-2}$. The value of $\omega$ obtained seems quite reasonable. In rigid solutions where the luminescent molecule does not rotate, the torsional vibrations are the only depolarizing factor in the solution, immediately following excitation during thermal relaxation $\left(T^{\prime} \approx 4.7 \times 10^{-12} \mathrm{~s}\right)$.

We thank Professor A. Schmillen (University of Giessen) for his valuable remarks.

ponents parallel and perpendicular to the electric vector $\boldsymbol{E}$ of the excitation light, and $P$ is the degree of polarization.

[2] A. Jabłoński, Bull. Acad. Polon. Sci., Ser. sci. math., astr. phys. 8, 259 (1960).

[3] The fundamental fluorescence anisotropy $r_{\mathrm{f}}$ is given by the Perrin formula [4]

$$
r_{\mathrm{f}}=0.6 \cos ^{2} \alpha-0.2,
$$


where $\alpha$ denotes the angle between the emission and absorption dipoles. In the case considered, $\alpha=0$ and therefore $r_{\mathrm{f}}=0.4$, which is named the principal fluorescence anisotropy. The observed limiting fluorescence anisotropy $r_{0}$ is slightly lower than the principal fluorescence anisotropy (0.4).

[4] F. Perrin, Ann. Phys. Paris 12, 169 (1929)

[5] A. Cahen, J. Chim. Phys. 30, 420 (1933).

[6] A. Jabłoński, Acta Phys. Polon. 7, 15 (1938).

[7] P. Pesteil and M. Barbaron, J. Phys. Radium 237, 284 (1953).

[8] E. Laffite, Ann. physique 10,71 (1955).

[9] J. Grzywacz, Bull. Acad. Polon. Sci., Ser. sci. math., astr. phys. 6, 705 (1958).

[10] J. Heldt, Acta Phys. Polon. 30, 3 (1966).

[11] A. Kawski, P. Czyż, and J. Kukielski, Acta Phys. Polon. 31, 825 (1967).

[12] A. Kawski, P. Czyż, and B. Cieślak, Z. Naturforsch. 22 a, 1507 (1967).

[13] P. Czyż and A. Kawski, Bull. Acad. Polon. Sci., Ser. sci. math., astr. phys. 17, 591 (1969).
[14] R. K. Bauer, Acta Phys. Polon. 35, 101 (1969)

[15] A. Kawski, I. Weyna, Z. Kojro, and A. Kubicki, Z. Naturforsch. 38a, 1103 (1983)

[16] I. Gryczyński, Ch. Jung, and H. Kawska-Kubicka, Z. Naturforsch. 36 a, 680 (1981).

[17] A. Kawski, J. Kukielski, and J. Kamiński, Z. Naturforsch. 34a, 1066 (1979).

[18] A. Kawski, Z. Kojro, and M. Alicka, Z. Naturforsch. 35 a, 1197 (1980).

[19] A. Jabłoński, Acta Phys. Polon. 10, 33, 193 (1950).

[20] C. Bojarski, Bull. Acad. Polon. Sci., Ser. sci. math., astr. phys. 6, 713 (1958).

[21] A. Jabłoński, Bull. Acad. Polon. Sci., Ser. sci. math., astr. phys. 16,601 (1968).

[22] A quantum-mechanical theory of the torsional oscillator is given by Hanus [23].

[23] W. Hanus, Acta Phys. Polon. 10, 173 (1951).

[24] A. Kawski, Z. Kojro, and A. Kubicki, Z. Naturforsch. in press. 